\title{
A neutron-scattering investigation of the magnetic structure and magnetic excitations in nanocrystalline $\mathrm{Tb}$
}

\author{
C. Vecchini and O. Moze ${ }^{a)}$ \\ CNR-INFM S3, Dipartimento di Fisica, Università di Modena e Reggio Emilia, Modena 41100, Italy
}

\author{
A. Hoser and M. Prager \\ Institut für FestkoerperForschung, Forschungzentrum Jülich, D-52425 Jülich, Germany \\ D. Argyriou \\ Berlin Neutron Scattering Center, Hahn Meitner Institut, D-14169 Berlin, Germany
}

\begin{abstract}
A. Michels
Paul Scherrer Institute, 5232 Villigen, Switzerland and Technische Physik, Universität des Saarlandes, Saarbrücken, Germany

\section{B. Roessli}

Laboratory for Neutron Scattering, ETHZ, Zurich, Switzerland and Paul Scherrer Institute, 5232 Villigen, Switzerland

\author{
J. Weissmüller, C. Lemier, and R. N. Viswanath \\ Institut für Nanotechnologie, Forschungzentrum Karlsruhe, D-76021 Karlsruhe, Germany
}

(Presented on 1 November 2005; published online 19 April 2006)

\begin{abstract}
The magnetic structure and magnetic excitations in nanocrystalline $\mathrm{Tb}$ have been investigated by neutron diffraction and neutron spectroscopy. This is a report on the long-range magnetic order and the magnetic excitations in a nanocrystalline elemental rare earth. Refinement of the neutron-diffraction data reveals an "average" magnetic structure of each crystallite which contains a significant out-of-plane component to the magnetic moment as well as a suppression of the high-temperature antiferromagnetic phase observed for coarse-grained $\mathrm{Tb}$. The inelastic-neutron-scattering measurements reveal the presence of a magnetic excitation of approximately $10 \mathrm{meV}$ at $2.5 \mathrm{~K}$. The excitation energy decreases with increasing temperature. The origins of this excitation are discussed with particular reference to the magnetic modes at the zone center observed for single-crystal Tb. (C) 2006 American Institute of Physics.
\end{abstract}

[DOI: $10.1063 / 1.2165139$ ]

\section{INTRODUCTION}

Crystalline rare earths have been widely studied in the past for their unique magnetic properties. Preparation techniques now make it possible to obtain specimens with grain sizes of a few nanometers, allowing for investigation of interesting effects due to the concomitant reduced dimensionality. Nanocrystalline materials have received enormous interest attention because of their remarkably different magnetic properties when compared to their coarse grain $(\mathrm{cg})$ counterparts. One of the most widely investigated elemental rare earths, $\mathrm{Tb}$, displays two different magnetic transitions at temperatures of 218 and $228 \mathrm{~K}$, respectively: the first a transition from a low-temperature basal plane ferromagneticspiral antiferromagnetic magnetic structure and the second an antiferromagnetic spiral-paramagnetic transition.

The magnetic microstructure of nanocrystalline (nc) $\mathrm{Tb}$ with a grain size of $12 \mathrm{~nm}$ has been recently investigated by small-angle neutron scattering. ${ }^{1}$ This study has clearly shown that the microscopic magnetic structure is highly inhomogeneous and that the high-temperature antiferromagnetic spiral structure is suppressed. In addition, the ferromagnetic ordering temperature was reported as $229 \mathrm{~K}$, higher than that for

${ }^{a)}$ Electronic mail: moze.oscar@unimo.it corresponding cg material. An enhanced magnetic anisotropy and correspondingly enhanced coercivity were also observed.

Neutron scattering has been used in the recent past to determine the magnetic structure, ${ }^{2}$ lattice dynamics, ${ }^{3}$ and magnetic excitations ${ }^{4-7}$ for $\mathrm{cg} \mathrm{Tb}$. The behavior of the magnetic excitations under pressure was reported more recently by Kawano et al. ${ }^{8}$ Two important parameters used in modeling the magnetic behavior for $\mathrm{nc} \mathrm{Tb}$ (Ref. 1) are the effective spin-wave stiffness constant and the anisotropy gap, which reflects dipolar and anisotropy contributions. From inelasticneutron-scattering (INS) results reported for single-crystal $\mathrm{Tb},{ }^{4-7}$ a high-energy excitation gap (which corresponds to an optical zone center magnon mode) in zero applied magnetic field lies at approximately $10 \mathrm{meV}$, while the anisotropy gap is about $1.7 \mathrm{meV}$, at $4.2 \mathrm{~K}$. A part of the present study is aimed at investigating the energy range of magnetic excitations in $\mathrm{nc} \mathrm{Tb}$ up to $20 \mathrm{meV}$ in order to determine the exact energy range in which they are present.

This work is meant to highlight the different behavior of this rare earth when in the form of a dense polycrystalline aggregate with a nanometer grain size with neighboring grains exchange coupled across metallic grain boundaries. Presented are (i) neutron-diffraction data which verify the suppression of the antiferromagnetic phase and the magni- 
tude and orientation of the magnetic moments and (ii) INS data which reveal the presence of the high-energy excitation gap. This is the first reported investigation of the ordered magnetic structure and dynamics in any elemental rare earth by neutron diffraction and INS, respectively.

\section{EXPERIMENT}

Several nc pellets of pure $\mathrm{Tb}$, each of approximately $8 \mathrm{~mm}$ diameter and $0.9 \mathrm{~mm}$ width, were prepared by the inert gas condensation technique at the Institut für Nanotechnologie, Forschungzentrum Karlsruhe, Germany using commercial grade $\mathrm{Tb}(99.8 \%)$. Coarse $\mathrm{Tb}$ was inserted in a controlled ultrahigh-vacuum atmosphere and heated up to the melting point. A continuous flow of inert He gas was used to collect the $\mathrm{Tb}$ nanoparticles which evaporate on a rotating cylinder, cooled by liquid $\mathrm{N}_{2}$, in the center of the vacuum chamber. The resulting material was then collected with a funnel in a sample holder and then compressed under high pressure in order to minimize oxidation of the surface and eventual contamination of the sample by oxygen. The sample was then polished and stored in a controlled Ar atmosphere. Due to the nanosize of the crystallites, the sample is very reactive with air but, after the treatment indicated above, no oxidation occurs inside the bulk of the sample even after months of exposure to air. A total of $1 \mathrm{~g}$ of $\mathrm{nc}$ Tb was produced, with a mean grain size of $12 \mathrm{~nm}$, as calculated from the integral width of the Bragg reflections of $\mathrm{x}$-raydiffraction spectra using the formalism of Klug and Alexander. ${ }^{9}$ Magnetization measurements confirm an ordering temperature of $227 \mathrm{~K}$, in agreement with the bulk magnetic properties reported previously for $\mathrm{nc} \mathrm{Tb}^{1,10-12}$

Neutron-diffraction measurements were performed on the E9 diffractometer located at the Hahn Meitner Institut, BENSC, Berlin, Germany with an incident wavelength of $\lambda=1.797 \AA$. Scans were performed at temperatures above $(250 \mathrm{~K})$ and below $T_{C}(220,200,175$, and $18 \mathrm{~K})$.

Inelastic-neutron-scattering measurements were performed using triple-axis and time-of-flight (TOF) techniques. A first attempt to locate an inelastic signal was performed at PSI (Villigen, Switzerland) on the triple-axis spectrometer (TASP). This measurement yielded evidence of a peak at an energy transfer of approximately $7.3 \mathrm{meV}$ at $200 \mathrm{~K}$. INS data were then collected on the triple-axis spectrometer UNIDAS (located at the Forschungzentrum Juelich, Germany) with a fixed initial neutron energy of $14.7 \mathrm{meV}$ for energy transfers of up to $12 \mathrm{meV}$. Inelastic scans were performed around the (101) magnetic reciprocal-lattice point. TOF measurements, which yield a more efficient and quicker coverage of the wave vector and energy range of the excitations, were collected on the SV29 spectrometer (also located at the Forschungzentrum Juelich) with incident neutron energies of $25 \mathrm{meV}$, in the temperature range of $2.5-250 \mathrm{~K}$.

\section{RESULTS AND DISCUSSION}

Analysis of the results is based on the standard master formula for the inelastic-neutron-scattering cross section from magnetic species:

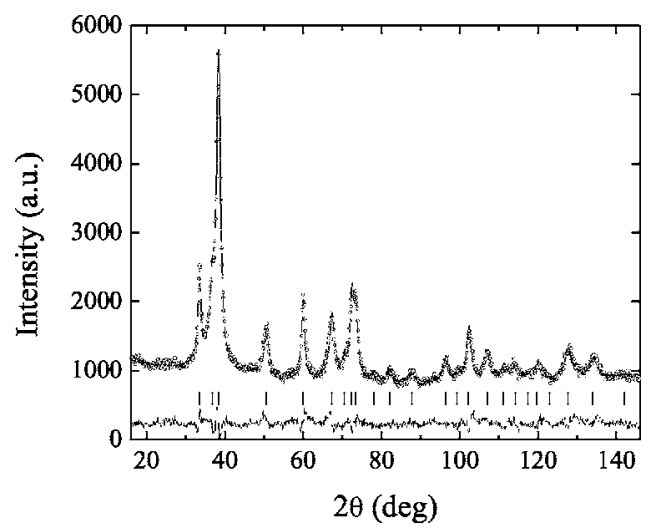

FIG. 1. Observed, refined, and difference neutron-diffraction pattern for nc $\mathrm{Tb}$ at $18 \mathrm{~K}$. The calculated peak positions are marked with vertical bars.

$$
\frac{d^{2} \sigma}{d \Omega d E} \propto \frac{k_{f}}{k_{i}}|f(Q)|^{2} S(Q, \omega),
$$

where $k_{f}$ and $k_{i}$ are final and initial neutron wave vectors, $Q=k_{f}-k_{i}$ the scattering vector, $f(Q)$ the magnetic form factor, $\omega$ the energy transfer between initial $E_{i}$ and final $E_{f}$ neutron energy states, $\omega=E_{i}-E_{f}$, and $S(Q, \omega)$ the scattering law, which is related to the imaginary part of the dynamic magnetic susceptibility $\chi^{\prime \prime}(Q, \omega), \quad S(Q, \omega)=\chi^{\prime \prime}(Q, \omega) /$ $\left[1-\exp (-\omega) / k_{B} T\right]$ with $k_{B}$ and $T$ Boltzmann's constant and temperature, in $\mathrm{K}$, respectively.

For a collinear ferromagnet, the elastic-neutronscattering cross section at a magnetic reciprocal-lattice point is

$$
\frac{d \sigma}{d \Omega} \propto|f(Q)|^{2} \sum_{i, j}\left\langle g J_{\perp}\right\rangle^{2} \exp \left[i Q \cdot\left(\mathbf{R}_{i}-\mathbf{R}_{j}\right)\right],
$$

where the summation is carried over atoms at positions $R_{i}$ and $R_{j}$ in the magnetic unit cell, $J$ the total angular momentum, $g J_{\perp}$ the component of the $\mathrm{Tb}$ magnetic moment perpendicular to the scattering vector, and $g$ the Landé factor. For the polycrystalline aggregate of $\mathrm{Tb}$ nanocrystals under consideration here, only the moment directions and magnitude with respect to the unique hexagonal $c$ axis can be determined. ${ }^{13}$

\section{A. Neutron diffraction}

Analysis of the neutron-diffraction data proceeded along the following lines: the crystal and magnetic structures were assumed identical to that for $\mathrm{cg} \mathrm{Tb}$, crystal space group $\mathrm{PG}_{3} / \mathrm{mmc}$ with two atoms per unit cell at $\left(\frac{1}{3}, \frac{2}{3}, \frac{1}{4}\right)$ and $\left(\frac{2}{2}, \frac{1}{3}, \frac{1}{4}\right)$ and a low-temperature magnetic structure with moments aligned in the basal plane. ${ }^{2}$ In addition to lattice constants, an overall temperature factor and an intrinsic line shape due to the nanosized Tb crystallites were refined. For temperatures below the ordering point, no antiferromagnetic Bragg peaks were observed in the temperature range where $\mathrm{cg} \mathrm{Tb}$ is in the spiral antiferromagnetic phase, thus confirming the suppression of this magnetic transition for $\mathrm{nc} \mathrm{Tb}^{1}{ }^{1}$ The lattice constants at $293 \mathrm{~K}$ are $a=3.5982 \AA$ and $c$ $=5.6960 \AA$ A Displayed in Fig. 1 is the observed and refined neutron-diffraction pattern at $18 \mathrm{~K}$. The refined moment at $18 \mathrm{~K}$ is found to be anomalously low, about $6.4 \mu_{B}$ which is 


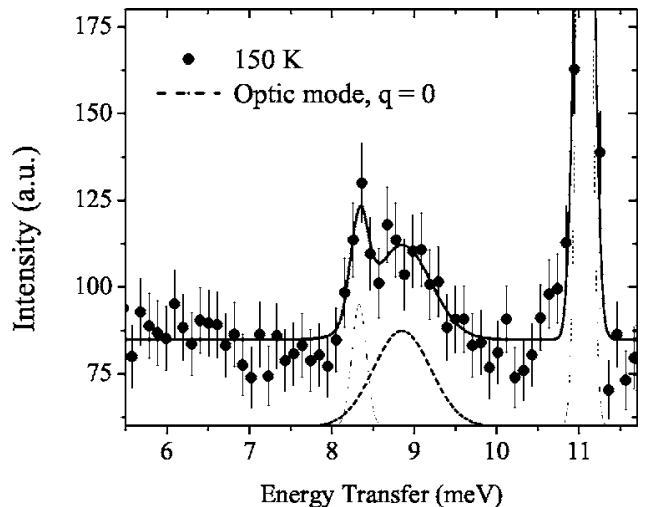

FIG. 2. Inelastic-neutron-scattering spectrum for $\mathrm{nc} \mathrm{Tb}$ at $150 \mathrm{~K}$ collected on the triple-axis spectrometer UNIDAS. Peaks at 8.3 and $11 \mathrm{meV}$ are due to instrumental effects. The peak at approximately $9 \mathrm{meV}$ is the intrinsic optic zone center (i.e., $\mathbf{q}=0$ ) magnon mode for $\mathrm{nc} \mathrm{Tb}$.

approximately $2 / 3$ of the value observed for coarse $\mathrm{Tb}$. At the present moment in time we have no convincing explanation for these two effects. High-field magnetization measurements of the saturation magnetization are planned in order to compare moment values with those obtained from neutron diffraction. A speculative suggestion is that the local crystalline electric field created by strain and defects in the nanocrystalline material be significantly large enough to give rise to a reduced ferromagnetic moment. More detailed neutrondiffraction experiments are presently underway to determine the dependence of the $\mathrm{Tb}$ moment over an extensive temperature range.

\section{B. Inelastic neutron scattering}

Displayed in Fig. 2 is the INS spectrum collected on UNIDAS at $150 \mathrm{~K}$. A broad intrinsic inelastic peak centered at $8.8 \mathrm{meV}$ is evident together with two peaks due to instrumental Bragg reflections at 8.3 and $11 \mathrm{meV}$. The TOF data reveal a peak at $100 \mathrm{~K}$ at an energy transfer of approximately $9 \mathrm{meV}$. These results are in accord with those for singlecrystal $\mathrm{Tb},{ }^{4,7}$ which report a zone center optic magnon mode at the same energy transfers. As seen from Fig. 3, the inelastic peak has moved at $2.5 \mathrm{~K}$ to a higher energy, nominally at just over $10 \mathrm{meV}$. The observed inelastic peak is thus most probably magnetic in origin and is tentatively assigned as the optic magnon mode.

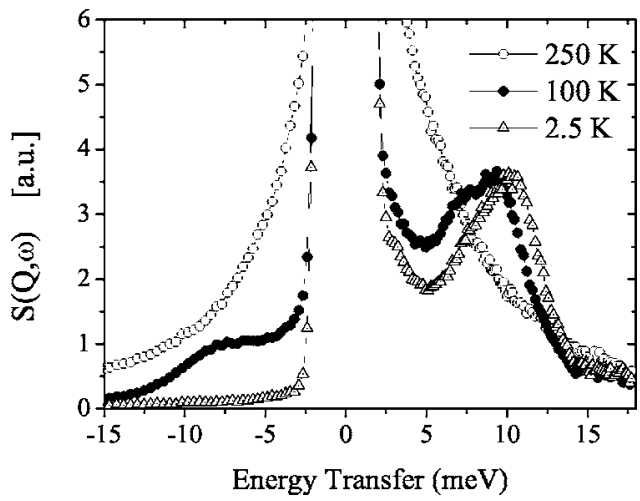

FIG. 3. The magnetic scattering law $S(Q, \omega)$ for $\mathrm{nc} T \mathrm{~Tb}$ at 250,100 , and $2.5 \mathrm{~K}$, as measured on the TOF spectrometer SV29.
The energies for the zero wave vector $(\mathbf{q}=0)$ acoustic and optical magnon modes for ferromagnetic $\mathrm{Tb}$ are, respectively, ${ }^{4-7,14-16}$

$E(0) \propto\left[-\left(2 P_{2} J^{2}+36 P_{6}{ }^{6} J^{6}\right)\right]^{1 / 2}$,

$E(0) \propto\left[-2 P_{2} J^{2}+4 \mathrm{I}^{\prime}(0) J^{2}\left(1-6 P_{6}{ }^{6} J^{6}\right)-36 P_{6}{ }^{6} J^{6}\right]^{1 / 2}$,

where $I^{\prime}(0)$ is the Fourier-transformed intrasublattice exchange interaction evaluated at a value of the wave vector $\mathbf{q}=0$ and $P_{n}{ }^{m}$ are anisotropy terms. Values for these terms with reference to single-crystal $\mathrm{Tb}$ are tabulated in Ref. 4 The pure anisotropy gap is given by Eq. (3) while Eq. (4) contains both anisotropy and exchange terms. For singlecrystal $\mathrm{Tb}$ at $90 \mathrm{~K}$, the energies of the acoustic and optic zone center branches, respectively, lie at 1.8 and $9 \mathrm{meV}$. Further measurements with different incident energies and a higher-energy resolution are presently underway in order to pick up all the important acoustic branches, which is expected to lie below $3-4 \mathrm{meV}$. Given that the intrinsic coercivity of $\mathrm{nc} \mathrm{Tb}$ is significantly larger than that for $\mathrm{cg} \mathrm{Tb}$, it will be of most interest to confirm if the anisotropy gap for $\mathrm{nc} \mathrm{Tb}$ is different from that of $\mathrm{cg} \mathrm{Tb}^{1,10-12}$

In summary, it can be concluded that (i) the magnetization in $\mathrm{nc} \mathrm{Tb}$ is reduced and the moments do not appear to lie totally in the basal plane but also have an out-of-plane component, and (ii) it is ferromagnetic for all temperatures up to the Curie point. The optical magnon mode energy at the zone center is of approximately the same energy as that of cg $\mathrm{Tb}$. There is no clear evidence of a pure anisotropy gap but higher-energy-resolved TOF measurements should identify and track the temperature dependence of this energy gap.

\section{ACKNOWLEDGMENTS}

Support from the European Commission under the Sixth Framework Programme through the Key Action: Strengthening the European Research Area, Research Infrastructures No. RII3-CT-2003-505925 (NMI3) and a MIUR postgraduate bursary for one of the authors (C.V.) are gratefully acknowledged.

${ }^{1}$ J. Weissmüller, A. Michels, D. Michels, A. Wiedenmann, C. E. Krill III, H. M. Sauer, and R. Birringer, Phys. Rev. B 69, 054402 (2004).

${ }^{2}$ O. W. Dietrich and J. Als-Nielsen, Phys. Rev. 162, 315 (1967).

${ }^{3}$ J. C. G. Houmann and R. M. Nicklow, Phys. Rev. B 1, 3943 (1970).

${ }^{4}$ H. Bjerrum Møller and J. C. G. Houmann, Phys. Rev. Lett. 16, 737 (1966).

${ }^{5}$ J. Jensen, J. C. G. Houmann, and H. Bjerrum Møller, Phys. Rev. B 12, 303 (1975).

${ }^{6}$ J. Jensen and J. W. Houmann, Phys. Rev. B 12, 320 (1975).

${ }^{7}$ J. C. G. Houmann, J. Jensen, and P. Touborg, Phys. Rev. B 12, 332 (1975).

${ }^{8}$ S. Kawano, J. A. Fernandez-Baca, and R. M. Nicklow, J. Appl. Phys. 75, 6060 (1994).

${ }^{9}$ H. P. Klug and L. E. Alexander, X-ray Diffraction Procedures for Polycrystalline and Amorphous Materials (Wiley, New York, 1974), Chap. 9.

${ }^{10}$ D. Johnson, P. Perera, and M. J. O’Shea, J. Appl. Phys. 79, 5299 (1996).

${ }^{11}$ M. J. O'Shea and P. Perera, J. Appl. Phys. 85, 4322 (1999).

${ }^{12}$ Z. C. Yan et al., Phys. Rev. B 67, 054403 (2003).

${ }^{13}$ G. Shirane, Acta Crystallogr. 12, 282 (1959).

${ }^{14}$ K. Niira, Phys. Rev. 117, 129 (1960).

${ }^{15}$ P.-A. Lindgård, A. Kowalska, and P. Laut, J. Phys. Chem. Solids 28, 1357 (1967).

${ }^{16}$ P.-A. Lindgård, Phys. Rev. B 17, 2348 (1978). 